\title{
Stellar clusters in dwarf galaxies ${ }^{\star}$
}

\author{
L. Vanzi ${ }^{1}$ and M. Sauvage ${ }^{2}$ \\ 1 European Southern Observatory, Alonso de Cordova 3107, Vitacura, Santiago, Chile \\ e-mail: lvanzi@eso.org \\ 2 CEA/DSM/DAPNIA/Service d'Astrophysique - UMR AIM, CE Saclay, 91191 Gif-sur-Yvette Cedex, France \\ e-mail: msauvage@cea.fr
}

Received 28 February 2005 / Accepted 13 October 2005

\section{ABSTRACT}

We present new observations in the $K_{\mathrm{s}}(2.2 \mu \mathrm{m})$ and $L^{\prime}(3.7 \mu \mathrm{m})$ infrared bands of a sample of blue dwarf galaxies with the larger aim of studying the population of massive stellar clusters, the occurrence of dust-embedded stellar clusters, and their properties. All $K_{\mathrm{s}}$ images show a rich population of clusters, but only a small fraction of them is bright in $L^{\prime}$. Most $L^{\prime}$ sources have radio counterparts. We derived the luminosity function in $K_{\mathrm{s}}$ for the galaxies IC 4661 and NGC 5408, finding both to be consistent with those of similar galaxies. We also compared the number of clusters and their luminosities with the star-formation rate of the host galaxies and found no compelling evidence of correlation. We conclude that young clusters and embedded clusters are a common feature of blue dwarf galaxies and possibly of galaxies in general, we suggest that their occurrence is due to purely statistical effects rather than a phenomenon related to specific physical conditions. In this sense we expect these objects to be abundant at high red-shift.

Key words. galaxies: dwarf - galaxies: starburst - galaxies: star clusters

\section{Introduction}

During the past years, it has been shown that star formation in massive stellar clusters can account for a large fraction of the star formation occurring in some galaxies. In particular, young massive clusters (YMC) with mass exceeding $10^{5} M_{\odot}$ have been found in a significant number of galaxies characterized by high star-formation rates (SFR), such as interacting and blue dwarf galaxies (BDG) (e.g. Whitmore et al. 1999; Cresci et al. 2004). Stellar clusters, especially when very young, can be obscured by large amounts of dust. Calzetti et al. (1997), for instance, found an anti-correlation between extinction and age in the five optically brightest young clusters observed in NGC 5253.

Infrared (IR) and radio observations have proved that dustembedded young clusters can be present not only in regular Star-Burst (SB) galaxies, but also in AGNs (Galliano et al. 2005), and even in the most metal-deficient blue compact galaxies (Thuan et al. 1999; Johnson et al. 2000; Turner \& Beck 2004). In some cases, a single cluster can account for a very large fraction, if not all of the IR emission of a galaxy. One example is the case of NGC 5253 where a single embedded cluster has an IR luminosity comparable to the optical-UV luminosity of the entire galaxy (Vanzi \& Sauvage 2004).

The existence of YMCs raises several interesting questions. Is star formation in massive clusters a common feature in

^ Based on observations obtained at the ESO VLT and ESO NTT; program 69.B-0345 and 71.B-0613. galaxies? What fraction of the total star formation in a galaxy occurs in clusters? How important can the extinction be, and therefore how much of the star formation is hidden in the optical-UV? Why do some galaxies host YMCs and others do not? And, in particular, why are deeply-embedded clusters observed in some cases and not in others? If YMCs originate, as it seems, in intense SB episodes (i.e. star-formation episodes occurring on large scales, short times, and with high efficiency), then we would expect them to be abundant in the early universe. It is then quite natural to ask how the phenomenon of YMCs changes in a pristine environment. We can study this issue in nearby dwarf star-burst galaxies, since these are, in many cases, the least chemically evolved objects available to us. With this objective in mind, we observed a sample of BDGs in the IR. Our purpose is to understand whether YMCs and obscured YMCs are a common feature of BDGs and what the conditions are under which they appear.

The present paper is organized as follows. In Sect. 2 we present the galaxy sample and the observations. In Sect. 3 we describe the population of stellar clusters, in Sect. 4 the embedded stellar clusters. In Sect. 5 we present a discussion of our results, and in Sect. 6 we summarize the conclusions of our work.

\section{Sample and observations}

The sample of galaxies was selected on the basis of the following criteria, i) firm detection in the IRAS bands; ii) evidence of 
Table 1. Properties of the sample galaxies observed. Column 1: name. Column 2: distance derived as described in the text. Column 3: $S F R$ in $M_{\odot} / \mathrm{yr}$ derived from the FIR. Column $4: K_{\mathrm{s}}$ total magnitude from this work, the major semiaxis in arcsec and the ellipticity of the $K_{\mathrm{s}} 3 \sigma$ elliptical isophote in parenthesis. Column 5: absolute $K_{\mathrm{s}}$ magnitude. Column 6: total number of sources detected. Column 7: sources brighter than -12. Column 8: sources brighter than -14. Column 9: fraction of luminosity due to the sources brighter than -12 . Column 10: fraction of luminosity due to sources brighter than -14 . Column 11: fraction of luminosity due to the most luminous cluster. Column 12: absolute magnitude of the host galaxy subtracted of the cluster contribution. The table is divided in two parts, the top showing the relatively compact and distant objects, and the bottom part the more extended and nearby galaxies.

\begin{tabular}{cccccccccccc}
\hline \hline Name & $d(\mathrm{Mpc})$ & $S F R$ & $K_{\mathrm{s}}$ & $M\left(K_{\mathrm{s}}\right)$ & $N_{*}$ & $N_{12}$ & $N_{14}$ & $L_{12} / L_{\text {tot }}$ & $L_{14} / L_{\text {tot }}$ & $L_{M} / L_{\text {tot }}$ & $M_{12}\left(K_{\mathrm{s}}\right)$ \\
\hline Tol 1924 & 41.1 & 0.58 & $12.6(11,0.6)$ & -20.5 & 27 & 27 & 21 & 0.60 & 0.59 & 0.07 & -19.5 \\
Tol 35 & 30.3 & 0.13 & $12.08(12,0.6)$ & -20.3 & 9 & 9 & 9 & 0.10 & 0.10 & 0.02 & -20.2 \\
Pox 36 & 15.9 & 0.05 & $12.63(13,0.5)$ & -18.4 & 48 & 37 & 1 & 0.24 & 0.02 & 0.02 & -18.1 \\
UM 462 & 15.3 & 0.05 & $13.55(7,0.5)$ & -17.4 & 17 & 12 & 1 & 0.26 & 0.05 & 0.05 & -17.1 \\
He 2-10 & 12.5 & 0.88 & $9.05(26,0.1)$ & -21.4 & 29 & 29 & 26 & 0.59 & 0.59 & 0.05 & -20.4 \\
II Zw 40 & 11.3 & 0.19 & $12.11(13,0.5)$ & -18.2 & 11 & 7 & 2 & 0.12 & 0.09 & 0.06 & -18.0 \\
Tol 3 & 10.2 & 0.12 & $10.76(16,0.4)$ & -19.2 & 35 & 27 & 9 & 0.19 & 0.11 & 0.02 & -19.0 \\
NGC 1705 & 9.0 & 0.03 & $10.5(18,0.2)$ & -19.3 & 40 & 18 & 1 & 0.16 & 0.12 & 0.12 & -19.1 \\
\hline NGC 5408 & 7.3 & 0.03 & $10.58(41,0.4)$ & -18.7 & 396 & 33 & 5 & 0.39 & 0.26 & 0.12 & -18.2 \\
IC 4662 & 4.4 & 0.04 & $9.90(31,0.3)$ & -18.3 & 500 & 14 & 1 & 0.09 & 0.02 & 0.02 & -18.2 \\
NGC 5253 & 3.3 & 0.07 & $9.01(40,0.5)$ & -18.6 & 594 & 16 & 1 & 0.08 & 0.02 & 0.02 & -18.5 \\
\hline
\end{tabular}

massive clusters from previous imaging studies; iii) evidence of intense star-formation. The sample of galaxies is presented in Table 1. It is divided in two sections: i) relatively compact and distant galaxies where only a few bright sources are detected (upper section), and ii) more extended and nearby galaxies where a large number of sources are detected (lower section). The distances were calculated assuming $H_{0}=70 \mathrm{~km} \mathrm{~s}^{-1} \mathrm{Mpc}^{-1}$ except for the galaxy NGC 5253, where we use the value of Gibson et al. (2000), and for the galaxies Tol 35, Tol 3 (NGC 3125) and, UM 462, where we use the values of Vanzi et al. (2002). In Table 1 we also report the SFR derived from the 60 and $100 \mu \mathrm{m}$ IRAS fluxes using the expressions given by Condon et al. (1991) and Kennicutt (1998).

A detailed analysis of NGC 5253 was already presented in our previous papers, Vanzi \& Sauvage (2004) and Cresci et al. (2005). However this galaxy was included in the present discussion both for completeness and because it is relevant as a comparison for the other objects.

Images in $K_{\mathrm{s}}(2.2 \mu \mathrm{m})$ and $L^{\prime}(3.7 \mu \mathrm{m})$ were obtained with ISAAC at the ESO VLT in service mode during periods 69 and 71 for all galaxies but NGC 1705. ISAAC is equipped with a $1024 \times 1024$ Hawaii detector for short wavelengths (1-2.5 $\mu \mathrm{m})$, and with a $1024 \times 1024$ Aladdin detector for long wavelengths $(2.5-5 \mu \mathrm{m})$. The scale is $0.147 \mathrm{arcsec} / \mathrm{pix}$ in the first case and $0.07 \mathrm{arcsec} / \mathrm{pix}$ in the second case. For the $K_{\mathrm{s}}$ images, we jittered ON source in the case of the compact objects (upper section of Table 1), while the ON-OFF technique was applied for the largest galaxies. In $L^{\prime}$ we chopped in all cases with a throw of 30 arcsec. The total integration time on source was 5 min per galaxy in $K_{\mathrm{s}}$ and about $40 \mathrm{~min}$ in $L^{\prime}$. The seeing during the observations ranged from excellent $(0.4$ arcsec $)$ in the case of Pox 36, Tol 35, Tol 3, He 2-10, and UM 462, to good (0.7-0.8 arcsec) for IC 4662, NGC 5408, Tol 1924-416, and II $\mathrm{Zw} 40$, so that a significant improvement in the image quality was obtained with respect to the data already published for some of the galaxies in the sample. NGC 1705 was observed in $K_{\mathrm{s}}$ with SOFI at the ESO-NTT in December 1998. SOFI has a scale of $0.287 \mathrm{arcsec} /$ pix and it is equipped with a $1024 \times$ 1024 Hawaii detector identical to ISAAC. The sky was acquired OFF source, and the total integration time ON source was $24 \mathrm{~min}$. The seeing was about 0.7 arcsec during the observations. The data reduction followed the standard steps of flat-fielding, background subtraction, and co-adding, and it was performed with IRAF.

\section{The population of stellar clusters}

We used the $K_{\mathrm{s}}$ images to study the cluster population of the sample galaxies. In Figs. 1 and 2 the $K_{\mathrm{s}}$ images of the galaxies observed are shown. The closest galaxies - IC 4662, NGC 5408, and NGC 5253 - are quite extended and cover, or even exceed, the field of view of ISAAC. They are resolved in a large number of compact sources. The other galaxies fit well in the 2.5 arcmin field of ISAAC, 5 arcmin for NGC 1705 observed with SOFI, and show a limited number of resolved objects. We used "daofind" in IRAF with a $5 \sigma$ threshold limit to identify compact sources in the images. Aperture photometry was extracted for all sources identified in the fields. Even in the cases of excellent seeing, we used an aperture with a radius of 0.8 arcsec, since many of the detected sources are resolved and extended. The background was measured around each object in an annulus of 1 arcsec radius and 2 arcsec width and then subtracted. We focussed on the sources that are brighter than $M_{K}=-12$, considering this value as an upper limit for stellar luminosities (Humphreys 1983). We can safely assume that all sources brighter than this limit are stellar clusters. The sources that were obviously not related to the target galaxy, because they are too bright or too far from it, were considered foreground stars and discarded from the analysis. This, of course, does not rule out contamination from foreground stars; 


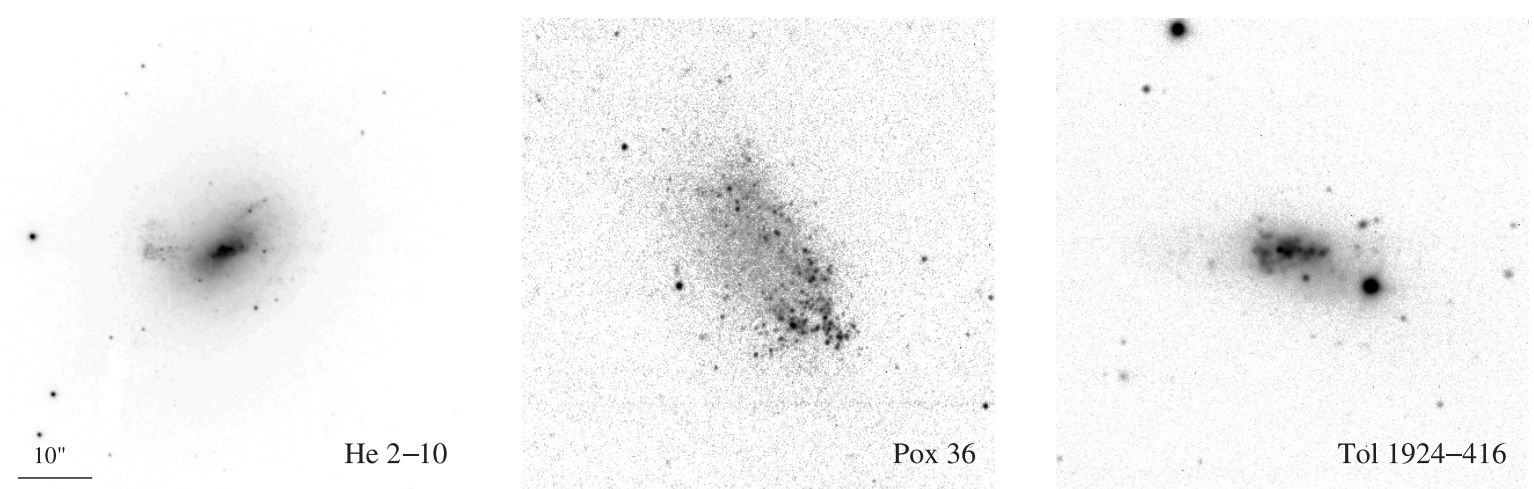

Tol 3
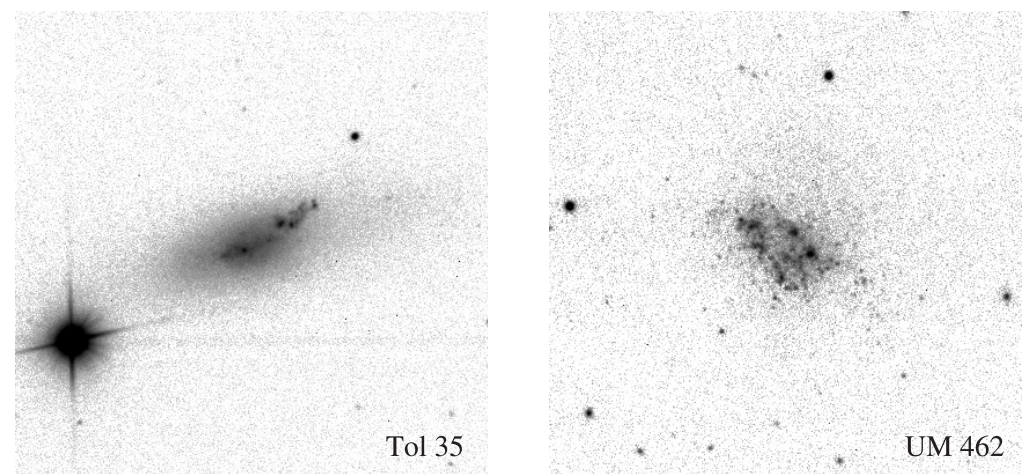

Fig. 1. Images in $K_{\mathrm{s}}$ band displayed in logarithmic scale. The field of view is 60 arcsec. North is up, East to the left.
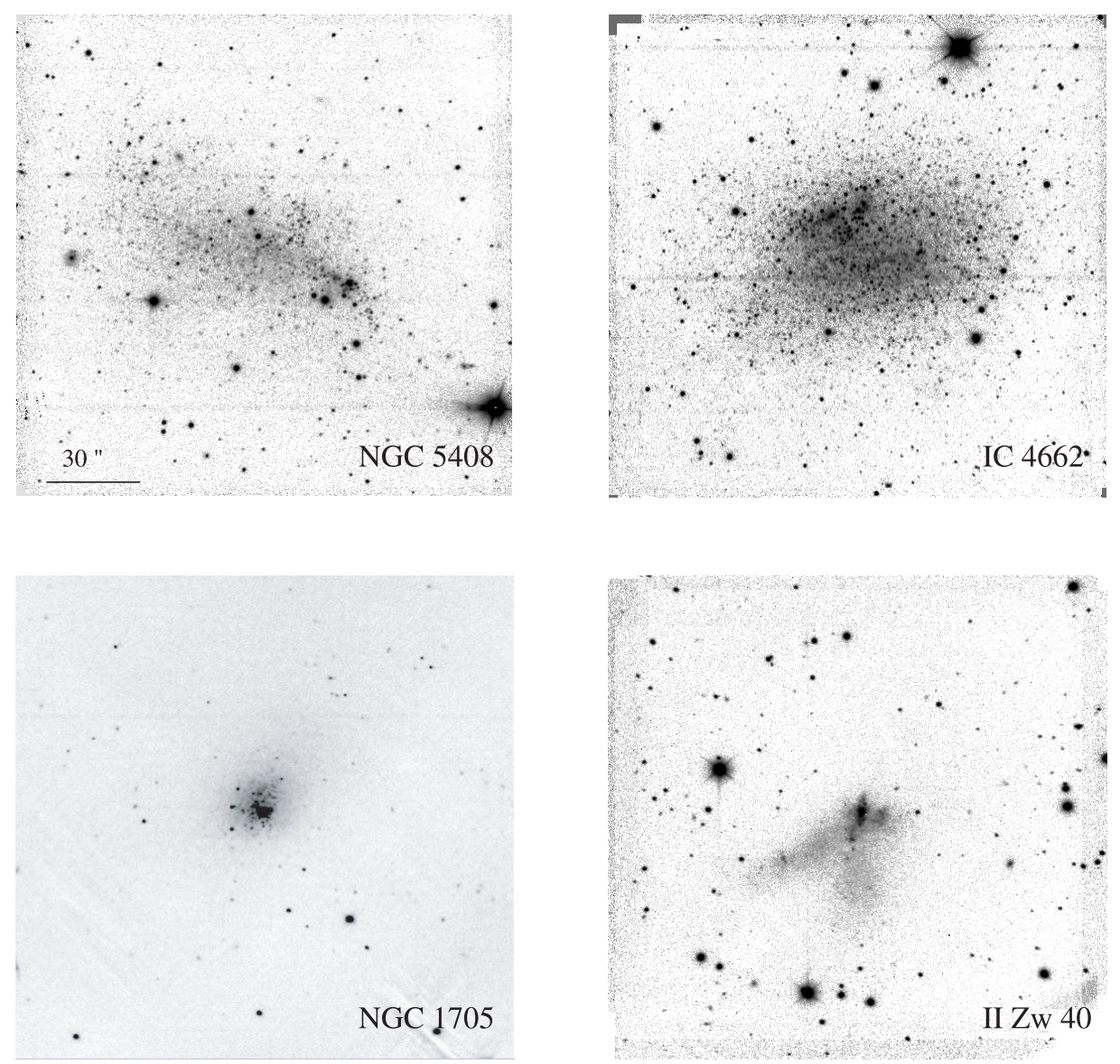

Fig. 2. Images in $K_{\mathrm{s}}$ band displayed in logarithmic scale. The field of view is 160 arcsec. North is up, East to the left. 
however, we expect this contamination to be negligible. The total number of sources identified in the fields and the number of sources brighter than $M_{K}=-12$ are reported in Table 1. We regarded the clusters with $M_{K}<-14$ as exceptionally bright, and their number is also listed in Table 1.

We measured the $K_{\mathrm{s}}$ total magnitude of the galaxies by fitting elliptical isophotes and considering the flux comprised within the isophote with an average value of $3 \sigma$ above the background. This method worked quite well for most of the objects in our sample, and only has limitations in few cases. The irregular shape of II Zw 40, for instance, cannot be properly fitted by ellipses. NGC 5408 and IC 4662 are mostly resolved in compact sources and have only a very faint diffuse component, the discontinuous aspect of the images making the fitting process difficult. The total $K_{\mathrm{s}}$ fluxes, the major semiaxis, and the derived ellipticities are reported in Table 1. The typical errors on the total magnitudes are smaller than $0.05 \mathrm{mag}$. and are mostly due to the uncertainty on the background. The values obtained agree well with the 2MASS total fluxes reported in NED.

\subsection{Cluster counts}

As a first step in our analysis, we studied the occurrence of the clusters and the correlation of their number with other properties of the host galaxies, the SFR in particular. From the examination of Table 1, we notice that the number of detected bright clusters $\left(N_{12}\right)$ is in the same range for all galaxies, i.e. a few to few tens. The total number of sources detected $\left(N_{*}\right)$, however, shows a sharp jump for NGC 5408, IC 4662, and NGC 5253, with about 10 times as many objects detected as in the other galaxies. This can be explained because these galaxies are mostly resolved due to their distance. It is then reasonable to assume that all galaxies have similar cluster populations. This issue will be discussed further in the next section. Some confusion, however, is introduced by the uncertainty on the distances. For NGC 1705, for instance, Tosi et al. (2001) find a distance of 5.1 Mpc. If this were confirmed, it would probably point to an intrinsic difference in the number of sources detected in this object with respect to other galaxies in the same distance range. The most striking aspect, however, is the number of very bright clusters $\left(N_{14}\right)$ since it shows large differences from one galaxy to another. While most galaxies of our sample host typically only one, or very few clusters above the -14 threshold, in Tol 1924-416, Tol 35, Tol 3, and He 2-10, a large fraction of (if not all) bright clusters detected are more luminous than -14 .

Possibly, the comparison makes more sense when normalized quantities are considered. We used the number of clusters and the SFR normalized to the absolute $K_{\mathrm{S}}$ magnitude of the host galaxies. For the number of clusters, we used an expression similar to the one used by Östlin (2000), $S=$ $N \times 10^{0.4\left(M_{K}+15\right)}$ which defines the specific frequency of clusters. With a similar expression we can normalize the SFR, $N S F R=S F R \times 10^{0.4\left(M_{K}+20\right)}$. The total luminosity of the host galaxy receives a large contribution from the clusters themselves. Therefore, we used the total $K_{\mathrm{s}}$ magnitude subtracted of the contribution of the clusters as normalization factor.

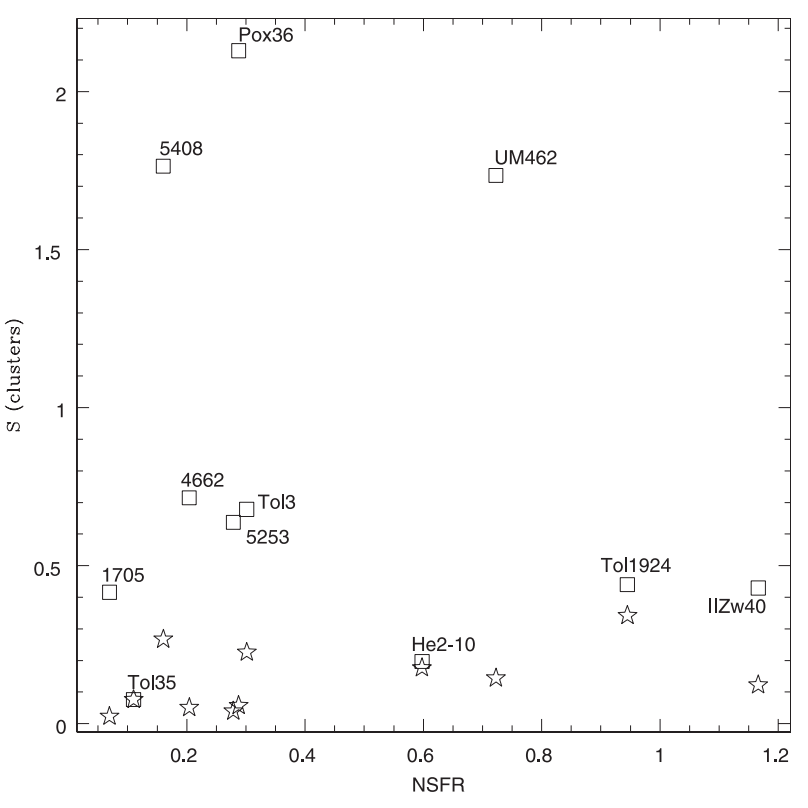

Fig. 3. Number of clusters brighter than -12 (squares) and brighter than -14 (stars), versus the IR SFR. Both quantities are normalized to the total $K_{\mathrm{s}}$ luminosity of the host galaxies subtracted of the clusters contribution.

The fraction of the luminosity provided by the clusters and the galaxies' absolute luminosities corrected for the clusters contribution are listed in Table 1. Indeed, one sees that in some cases the correction can be as large as 1 magnitude. Using the $K_{\mathrm{s}}$ luminosity of the host galaxy as the normalization factor is justified by the fact that this quantity is representative of the mass of the galaxy, since it is dominated by the bulk of old low luminosity stars. In the case of BDGs, however, we must consider that the population of evolved stars could be limited and that the $K_{\mathrm{s}}$ luminosity could only give a partial representation of the mass; despite this the $K_{\mathrm{s}}$ luminosity subtracted the contribution of the clusters remains our best representation of the mass of the galaxies. The range spanned is not very large, with a factor of about 20 between the most and the less luminous object. In Fig. 3 the normalized number of clusters $S$ is plotted versus NSFR: no clear correlation is found for the clusters brighter than -12 or for those brighter than -14 .

\subsection{Cluster luminosities}

We compared the luminosity of the clusters with the normalized SFR of the host galaxies. We used the expression of Harris (1991) and Larsen \& Richtler (2000) to calculate the specific $K_{\mathrm{s}}$ luminosity of the clusters, $T_{L}\left(K_{\mathrm{s}}\right)=100 \times L_{\mathrm{c}} / L_{\mathrm{t}}$, where $L_{\mathrm{t}}$ is the galaxy total luminosity and $L_{\mathrm{c}}$ is the luminosity of the clusters as in Larsen \& Richtler (2000). In the same expression we used the luminosity of the brigthest cluster, the integrated luminosity of all clusters brighter than $M_{K}=-12$, and the integrated luminosity of all clusters brighter than $M_{K}=-14$. Larsen \& Richtler (2000) found a correlation between the specific $U$-band luminosity of the clusters present in a galaxy and the SFR. We found a similar correlation in our sample of galaxies, when considering the brightest cluster only, as shown in the 


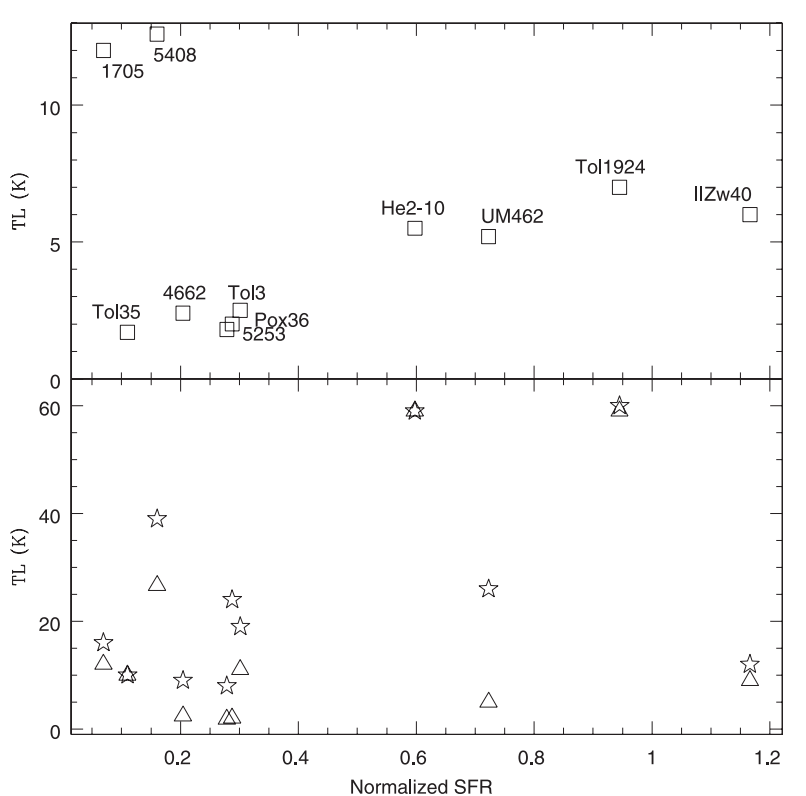

Fig. 4. The upper panel shows the specific $K_{\mathrm{s}}$ luminosity of the brightest cluster versus the normalized SFR of the host galaxy, and the lower panel the integrated specific $K_{\mathrm{s}}$ luminosity of the clusters brighter than -12 (stars) and brighter than -14 (triangles).

upper panel of Fig. 4. Both NGC 1705 and NGC 5408 obviously fall far from the trend defined by the other objects, they host a very bright cluster although their SFR is relatively low. In the lower panel of Fig. 4, we show the integrated luminosities of the clusters brighter than -12 (stars) and -14 (triangles): no correlation is found in this case.

For IC 4662, NGC 5408, and NGC 5253, the detected cluster population is large enough to derive the cluster luminosity functions. A detailed analysis of the luminosity function of NGC 5253 has been presented in Cresci et al. (2005), no further discussion of this galaxy will be presented here, except for comparison with the present results. The luminosity functions of IC 4662 and NGC 5408 are shown in Fig. 5. A completeness correction was calculated by adding to the images samples of 100 artificial sources uniformly distributed on a rectangular array of $10 \times 10$ points covering the galaxy area. Each sample included sources of the same brightness. The range from 16.0 to 20.0 was spanned in steps of $0.5 \mathrm{mag}$. The correction was then calculated by measuring the fraction of artificial sources recovered by our method for each given magnitude. The 50\% completeness limit is $m_{K}=19.0$. We fitted powerlaws to the completeness-corrected luminosity functions, using only the part located above the $50 \%$ completeness limit. We obtained -2.39 and -2.10 for IC 4662 and NGC 5408 respectively. These values agree with the value of -2.12 found by Whitmore et al. (1999) in the Antennae. The value found in NGC 5253 is -1.82 . Cresci et al. (2005) have shown that the luminosity function in $K$ is hardly affected by the extinction even in a case like NGC 5253, so that we expect our results to be quite robust from this point of view.

The $m_{K}=19.0$ completeness limit translates in the fact that our survey of clusters brighter than -14 is complete for all galaxies in the sample, while for the clusters brighter than -12 ,
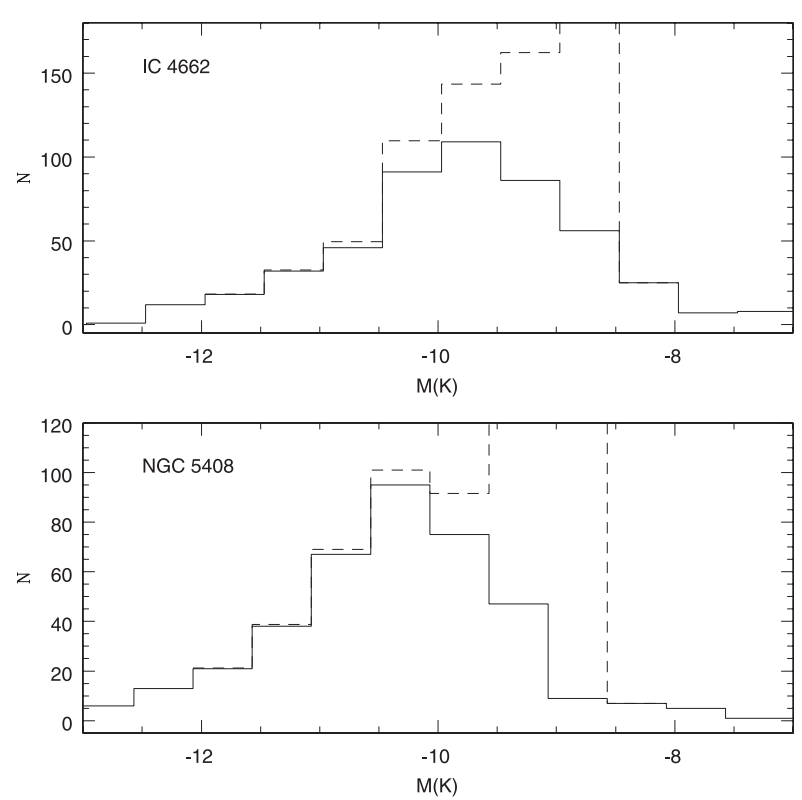

Fig. 5. The luminosity functions for the clusters in IC 4662 (top) and NGC 5408 (bottom), observed (solid), corrected for completeness (dashed).

it is complete only to a distance of about $15 \mathrm{Mpc}$. For this reason, Tol 1924-416 and Tol 35 must be considered with some care before being included in any statistical conclusion.

The luminosity functions also show that only a few tens of clusters are expected above the limit of $M_{K}=-11\left(m_{K}=19.0\right.$ at $10 \mathrm{Mpc}$ ), while their numbers rapidly increase for fainter magnitudes. Thus we expect a turnover in the total number of clusters detected in galaxies closer than about $10 \mathrm{Mpc}$, as is in fact observed.

\section{The embedded star clusters}

Only very few sources were detected in the $L^{\prime}$-band images, if we exclude the few obvious field stars. These sources are summarized in Table 2. The $L^{\prime}$-band sources detected in He 2-10 lead to a complete reappraisal of the star-burst in the galaxy, so we dedicated a separate paper to this object (Cabanac et al. 2005) and only report the brightest sources here. In addition to those listed in Table 2, one bright $L^{\prime}$ source has been recently observed in NGC 1705 by Cabanac (private communication). The total number of clusters brighter than -12 detected in $K_{\mathrm{s}}$ is 229 , of these only 12 have counterparts in $L^{\prime}$. The $L^{\prime}$ band detections are in the closest galaxies, so that there is a clear selection effect. Apart from this, there is no correlation between the number of $L^{\prime}$ sources detected and other properties of the galaxies.

Most sources detected in $L^{\prime}$ can be associated to compact radio sources. NGC 5253-5 is associated to the radio supernebula observed by Turner et al. (2000; see also Vanzi \& Sauvage 2004; Cresci et al. 2005; Turner et al. 2003, for discussion). The association of radio and $L^{\prime}$ sources in He 2-10, a key point for that object, is extensively discussed in Cabanac et al. (2005). Surprisingly, Johnson et al. (2003) do not find 
Table 2. $K_{\mathrm{s}}$ and $L$ photometry of the sources detected in the $L$ images. The data for NGC 5253 are from Vanzi \& Sauvage (2004), and for He 2-10 from Cabanac et al. (2005).

\begin{tabular}{ccccc}
\hline \hline Name & $K_{\mathrm{s}}$ & $L$ & $K-L$ & $M(L)$ \\
\hline Tol 3-1 & 14.81 & 13.62 & 1.19 & -16.42 \\
IC 4662-1 & 16.27 & 14.54 & 1.73 & -13.68 \\
IC 4662-2 & 15.55 & 14.83 & 0.72 & -13.39 \\
IC 4662-3 & 15.99 & 15.71 & 0.28 & -12.51 \\
IC 4662-4 & 15.85 & 15.63 & 0.22 & -12.59 \\
NGC 5408-1 & 17.06 & 14.09 & 2.97 & -15.23 \\
NGC 5253-5 & 13.46 & 9.07 & 4.39 & -18.52 \\
NGC 5253-5a & 15.54 & 12.51 & 3.03 & -15.08 \\
He 2-10-1 & 15.61 & 13.53 & 2.08 & -16.95 \\
He 2-10-4a & 13.92 & 13.04 & 0.88 & -17.44 \\
He 2-10-5 & 15.29 & 13.34 & 1.95 & -17.14 \\
\hline
\end{tabular}

any radio source that can be associated to the bright $L^{\prime}$ source detected in NGC 1705.

Among the faint $L^{\prime}$ sources, those detected in IC 4662 are remarkable for their radio counterparts: IC 4662-1 is coincident with the bright radio source detected by Johnson et al. (2003) and corresponds to the optically bright region called A1 by Hunter et al. (2001); IC 4662-4 can be associated to the fainter radio source to the north of region A1 (Johnson et al. 2003). Possible radio counterparts of IC 4662-2 and IC 4662-3 are less obvious, as both sources lie at the inner edges of the bright radio sources corresponding to the regions A1 and A2. The radio sources are thermal and powered by young and embedded massive clusters according to Johnson et al. (2003). NGC 5408-1 can be associated to the radio source A detected by Stevens et al. (2002), our $L^{\prime}$ source is elongated with a size of about 1 arcsec in the direction of the radio sources $\mathrm{B}$ and $\mathrm{C}$. Interestingly the radio sources B, C, and D (which we do not, or only partially detect in $L^{\prime}$ ) have negative spectral indexes that indicate a non thermal spectrum. This is probably due to SNe. The radio source $\mathrm{A}$, which is relatively bright, has a much flatter spectrum, indicating free-free emission and therefore a younger cluster.

Finally, the $L^{\prime}$ source named Tol 3-1 coincides, within less than 2 arcsec, with the radio source NGC 3125: a detected by Stevens et al. (2002), while we only detect a very faint diffuse emission in the position of the radio source NGC 3125:B. An extremely faint and diffuse component is visible in the $L^{\prime}$ images of Tol 1924 and Tol 35 in the position of the galaxy.

This exploration of the observed $L^{\prime}$ sources thus reveals a direct connection between the presence of IR excess in a cluster and the existence of a compact radio counterpart that often has a thermal spectrum. A similar association is also found in compact HII regions and is likely due to the same physics, only scaled-up. The very good correlation between radio and IR sources also shows how surveys in the IR are a viable alternative to the detection of embedded YMCs. We stress that all $L^{\prime}$ sources are also detected in $K_{\mathrm{s}}$, so that while $L^{\prime}$ only samples the youngest objects, $K_{\mathrm{s}}$ is sensitive to massive clusters starting from an age of few Myr.

\section{Discussion}

We do not have information that is complete enough to derive the properties (such as age and mass) of the clusters observed; however, we can certainly make a few general statements. From the behavior of the $K$ magnitude versus time calculated by Leitherer et al. (1999 - SB99), we notice that a coeval cluster of stars with a mass of $10^{6} M_{\odot}$ has a $K$ absolute magnitude above -14 only when it is younger than about $100 \mathrm{Myr}$, while it stays above -12 for more than $1 \mathrm{Gyr}$. We can assume $10^{6} M_{\odot}$ as an upper limit for the mass of the clusters. Most clusters with estimated masses are, in fact, in the $10^{5} M_{\odot}$ range or below. Therefore, we conclude that the brightest clusters we observed, those with absolute $K_{\mathrm{s}}$ magnitudes above -14 , are certainly younger than $100 \mathrm{Myr}$ and most likely younger than few tens Myr. The other clusters, those between -14 and -12 , can be either massive and relatively evolved $\left(\approx 10^{5} M_{\odot},>100 \mathrm{Myr}\right.$ ) or less massive and therefore younger. According to the mass function derived by Cresci et al. (2005), we expect very few $10^{6} M_{\odot}$ objects, while few tens of objects can easily be in the range $10^{4}-10^{5} M_{\odot}$. Assuming that the mass function of Cresci et al. also describes the galaxies in the current sample, it is likely that all observed clusters are younger than 100 Myr. To further support this idea, we can add that it is unlikely that clusters such as those observed are formed for a long period of time in a single galaxy. Based on their luminosities, we cannot exclude that a fraction of the clusters detected are old globulars. The "contamination" by globular clusters could possibly explain why the luminosity function of IC 4662 is somehow stepper than usual, while a lack of evolved clusters could explain the flatter behavior of NGC 5253.

From their luminosities and radio properties, we can assume that the $L^{\prime}$ sources presented in this work are young massive clusters still embedded in a dusty cocoon. Since dustembedded clusters are found equally in galaxies with different properties, we attribute their rarity to a short evolutive time scale rather than to peculiar conditions that are difficult to achieve. If we assume $100 \mathrm{Myr}$ as an upper limit for the age of the clusters observed and we consider all galaxies to the distance of He 2-10 for which the $L^{\prime}$ sample can be assumed complete, we find that the dusty phase of a cluster lasts less than $10 \mathrm{Myr}$, simply based on the occurrence of $L^{\prime}$ detections (one out of 12). By dusty phase, we mean here the epoch when the cluster has not yet cleared the surrounding area, and its spectral energy distribution is IR-dominated. This is supported by the behavior of the radio spectral index versus the $\mathrm{L}$ brightness, in the sense that the brightest $\mathrm{L}$ sources tend to have thermal radio spectra. The evolution of the radio spectral index with time has been quantified by Galliano et al. (2005), who find that radio indices above -0.6 imply ages below about 5 Myr. Since it takes a few Myr for a cluster to develop SNe, the IR bright phase of a cluster must be very short and must evolve quickly toward the optically bright phase. Kobulnicky \& Johnson (1999) suggested that the densest and most obscured phases of the SSC's precursors, observed by them in $\mathrm{He}$ 2-10 and characterized by rising radio spectra, may last only about $0.5 \mathrm{Myr}$. Similar sources have been observed by 
Beck et al. (2002) in II Zw 40. We support, therefore, a picture where the massive cluster precursors are only visible in the radio and where the dusty IR phase lasts only few Myr before the clusters become NIR and optically observable.

From the correlation between the specific $U$-band luminosity of the brightest clusters and the SFR, Larsen \& Richtler (2000) conclude that the formation of YMCs must be favored in environments with active star formation. We observe a similar correlation in $K_{\mathrm{s}}$; however, we notice that the same behavior would also arise if the most luminous clusters contribute significantly to the SFR of the galaxies, as measured from the FIR flux. This fact is certainly observed in NGC 5253. Therefore, the correlation claimed by Larsen \& Richtler (2000) could be partially (or even totally) biassed by the contribution of the brightest cluster itself to the SFR of the galaxy. If in the specific luminosity we include the contribution of fainter clusters, the correlation disappears, possibly because the less luminous clusters contribute more to the $K_{\mathrm{S}}$ luminosity than to the SFR. In addition, if we assume that clusters are formed following a common mass function in all galaxies, then galaxies with high SFR would be obviously advantaged with respect to the occurrence of YMCs for simple statistical reasons. The non linearity of the $M / L$ relation amplifies this effect. As for the luminosity of the most luminous clusters, two further effects must be considered: low number statistics and age. According to SB99 a coeval population of stars with solar metallicity spans almost 4 mag in $K$ luminosity and almost 3 mag in $U$ luminosity during the first $100 \mathrm{Myr}$ with a quite discontinuous behavior. Thus, it seems unlikely that clusters from different galaxies could produce a trend like the one observed by Larsen $\&$ Richtler (2000), because the simple age difference could easily produce a scatter of at least a factor 10 , as observed for NGC 1705 and IC 4662. To conclude on this issue, a correlation between the specific luminosity of the most massive cluster and the SFR of a galaxy is observed for most, but not all, galaxies. This relation seems to arise from a combination of observational effects and biasses, as described above, rather than from a common physical phenomenon. When normalized to the $K_{\mathrm{s}}$ luminosity of the host galaxy, no strong correlation is found between the number of clusters and the SFR.

Though we cannot exclude that clusters are preferentially formed in high density or high star-formation regions, as suggested by Larsen \& Richtler (2000), we propose an alternative picture that could complement or even replace the previous one. It is very well known from studies in the Galaxy that stars, and massive stars in particular, form in associations or clusters, so that star formation in clusters must be a common phenomenon. The population of young clusters in the Galaxy is limited, but in those galaxies where young clusters are abundant, the luminosity functions observed to date are consistent with each other. Though a dependency of the luminosity function on the properties of the host galaxy cannot be excluded, no elements have been collected so far in this direction. We can, therefore, assume that, at least in the range of SFR that we have explored, clusters are commonly formed in galaxies, possibly following a universal mass function. Galaxies with relatively low SFR would sample the low end of the luminosity function, while the most powerful SB would span the entire range of cluster luminosities. The main result of our work, therefore, can be stated in the following way. The presence of YMCs in a galaxy could be a purely statistical effect rather than a phenomenon related to particular physical conditions. If it is true that massive clusters are a normal product of star-formation and do not require special conditions to form, other than the high SFR needed to reach statistical significance, they must be abundant in high red-shift objects.

\section{Conclusions and summary}

We observed a sample of BDGs in the $K_{\mathrm{s}}(2.2 \mu \mathrm{m})$ and $L^{\prime}$ (3.7 $\mu \mathrm{m})$ IR bands. Our conclusions can be summarized as follows:

1. The observed galaxies proved to be extremely rich in bright sources in $K_{\mathrm{s}}$. Many of these sources are bright clusters $M_{K}<-12$, some show exceptionally high luminosity $M_{K}<-14$. We believe these clusters to be mostly young, typically younger than $100 \mathrm{Myr}$, and massive typically more than $10^{4} M_{\odot}$.

2. We did not find a compelling correlation between the number and luminosity of the clusters and the total SFR of the host galaxies.

3. For the two closest galaxies (IC 4662 and NGC 5408) it was possible to derive a cluster luminosity function that is described well by a power law with index -2.39 and -2.10 , respectively.

4. Only few clusters have $L^{\prime}$ counterparts. We interpret the lack of $L^{\prime}$ bright clusters as the result of a short evolutionary time scale of the early dusty phase. We calculated this dusty IR-dominated phase as lasting less than 10 Myr. All $L^{\prime}$ sources have radio counterparts with the exception of the one in NGC 1705.

5. We conclude that star formation in clusters and embedded clusters is a common feature in star-burst galaxies and possibly in galaxies in general. The formation of massive clusters does not require special conditions, and clusters may follow a common mass function in all galaxies. Since a high SFR enhances the occurrence of YMCs for statistical reasons, we expect them to be abundant at high red-shift.

Acknowledgements. This research has made use of the NASA/IPAC Extragalactic Database (NED) which is operated by the Jet Propulsion Laboratory, California Institute of Technology, under contract with the National Aeronautics and Space Administration. We wish to thank the Paranal ESO staff for carrying the observations used for this work in service mode. We are grateful to Kimberly Dopke and Francesco Vanzi for their careful reading of the manuscript, and to the anonymous referee whose comments were very valuable and improved this work considerably.

\section{References}

Beck, S. C., Turner, J. L., Langland-Shula, L. E., et al. 2002, AJ, 124, 2516

Cabanac, R., Vanzi, L., \& Sauvage, M. 2005 
Calzetti, D., Meurer, G. R., Bohlin, R. C., et al. 1997, AJ, 114, 1834

Condon, J. J., Anderson, M. L., \& Helou, G. 1991, ApJ, 376, 95

Cresci, G., Vanzi, L., \& Sauvage, M. 2005, A\&A, 443, 447

Galliano, E., Alloin, D., Pantin, E., Lagage, L. O., \& Marco, O. 2005, A\&A, 438, 803

Gibson, B. K., Stetson, P. B., Freedman, W. L., et al. 2000, ApJ, 529, 723

Harris, W. E. 1991, ARA\&A, 29, 543

Humphreys, R. M. 1983, ApJ, 269, 335

Hunter, D. A., et al. 2001, ApJ, 553, 121

Johnson, K. E., Leitherer, C., Vacca, W. D., \& Conti, P. S. 2000, AJ, 120,1273

Johnson, K. E., Indebetouw, R., \& Pisano, D. J. 2003, AJ, 126, 101

Kobulnicky, H. A., \& Johnson, K. E. 1999, ApJ, 527, 154
Kennicutt, R. C. 1998, ARA\&A, 36, 189

Larsen, S. S., \& Richtler, T. 2000, A\&A, 354, 836

Leitherer, C., Schaerer, D., Goldader, J. D., et al. 1999, ApJS, 123, 3

Östlin, G. 2000, in Massive Stellar Clusters, ASP Conf. Ser., 63

Stevens, I. R., Forbes, D. A., \& Norris, R. P. 2002, MNRAS, 335, 1079

Tosi, M., Sabbi, E., Bellanzini, M., \& Aloisi, A. 2001, AJ, 122, 1271

Thuan, T. X., Sauvage, M., \& Madden, S. 1999, ApJ, 516, 783

Turner, J. L., Beck, S. C., \& Ho, P. T. P. 2000, ApJ, 532, 109

Turner, J. L., Beck, S. C., Crosthwaite, L. P., \& Larkin, J. E. 2003, Nature, 423, 621

Turner, J. L., \& Beck, S. C. 2004, ApJ, 602, L85

Vanzi, L., \& Sauvage, M. 2004, A\&A, 415, 509

Vanzi, L., Hunt, L. K., \& Thuan, T. X. 2002, A\&A, 390, 481

Whitmore, B. C., Zhang, Q., Leitherer, C., et al. 1999, AJ, 118, 1551 